(C) 2011 IEEE. Personal use of this material is permitted. Permission from IEEE must be obtained for all other uses, in any current or future media, including reprinting/republishing this material for advertising or promotional purposes, creating new collective works, for resale or redistribution to servers or lists, or reuse of any copyrighted component of this work in other works. 


\title{
An Innovative Approach to Manage Prosumers in Smart Grid
}

\author{
A.J. Dinusha Rathnayaka, Vidyasagar M. Potdar, Samitha J. Kuruppu \\ Digital Ecosystems and Business Intelligence Institute \\ Curtin University of Technology \\ Perth, Western Australia \\ abekoon.rathnayaka@postgrad.curtin.edu.au
}

\begin{abstract}
Smart Grid (SG) achieves bidirectional energy and information flow between the energy user and the utility grid, allowing energy users not only to consume energy, but also to generate the energy and share with the utility grid or with other energy consumers. This type of energy user is called the "prosumer". The sustainability of the SG energy sharing process depends on its participating prosumers. Hence the prosumer management schemes are crucial within the energy sharing field. However, the existing literature on SG energy sharing has shown little attention on prosumer participation and management. The contribution of this paper is twofold. First we critically analyze the prosumer management schemes used by existing approaches and identify the open research issues. Second, we introduce a novel concept to manage the prosumers in the form of goaloriented virtual prosumer-communities and we discuss the aspects of prosumer-community formation, growth and overallmanagement. The main significance of this approach is that the prosumer-communities facilitate the prosumers with similar interest to join together and increase the quantity of energy to be auctioned to the SG and accordingly increase the bargaining power in the energy market. In addition, the prosumercommunities can attain more sustainable energy sharing process in long-term.
\end{abstract}

Keywords-smart grid; energy sharing; prosumer; prosumercommunity; bargaining power; sustainability

\section{INTRODUCTION}

The demand for energy in the world is continually rising. Worldwide energy consumption is expected to increase by 49 percent from 2007 to 2035[1]. The majority of the current demand is met by non-renewable energy sources like coal, petroleum, natural gas etc., but using such non-renewable energy sources contributes to greenhouse gas emissions and global warming. In today's world, the focus is more on shifting the energy usage of users from non-renewable sources to renewable sources of energy such as solar, water, and wind. Further, users are being encouraged not only to reduce their household energy consumption, but also to generate renewable energy and either to store the excess energy for future usage or to return the excess energy back to the utility grid. In order to achieve such a framework, the vision of SG has been proposed in literature. Smart Grid (SG) is defined as "an electricity network that can intelligently integrate the behaviour and actions of all users connected to it - generators, consumers and those that do both (prosumers) - in order to deliver sustainable, economic and secure electricity supplies" [2]. SG integrates ICT (Information Communication Technologies) with advanced power electronic technologies to achieve a bidirectional flow of electricity and information [3]. It also exhibits numerous desirable characteristics such as selfhealing, high reliability, increased efficiency, improved power quality, resistance to cyber attacks, and user friendliness [4].

In order for the SG energy sharing framework to work seamlessly, it requires the effective integration of different key elements namely; (i) smart devices, (ii) bi-directional communication, (iii) software infrastructure and (iv) dynamic prosumer base.

1) Smart devices: smart devices such as smart sensors/ monitors, smart storages, smart meters and smart gateways.

2) Bi-directional communication: the possible technologies would be fixed power line; wireless technologies [5]: WiFi, Zigbee, Z-Wave; and hybrid (combination of many technologies).

3) Software infrastructure: software applications to run the SG itself, and also to provide billing, consumer interface activities, etc.

4) Dynamic prosumer base: Even though the above factors are optimally integrated, the sustainability of energy sharing process is heavily dependent on its participating prosumers. For example, if a set of prosumers suddenly disagree to share the excess energy with the utility grid, in the worst-case the utility company may not be able to satisfy the entire consumer demand by itself. Therefore this element plays a critical role in achieving a sustainable energy sharing process.

The literature reveals that there are considerable amount of research has been done on development of smart device platforms, development of frameworks for enabling two-way communication, and development of software infrastructures[6] [7] [8]. However there has been little investigation in prosumer management schemes in existing SG energy sharing approaches. In existing approaches, the prosumers are participated in the energy sharing either as individual prosumers or simple prosumer-groups. However those methods are not very effective in addressing some vital issues like increasing prosumers' bargaining power in the 
energy market and achieving long-term sustainability. To date, in literature, no research has attempted to portray the overall position of managing prosumers in the contemporary SG research field or to identify the open research issues lagging in existing literature. In this paper, we first analyze the current schemes for managing prosumers in SG and highlight the research issues. Then we introduce a novel method to manage the prosumers in the form of goal-oriented virtual prosumercommunities. According to the existing body of literature, there is no published work on introducing a community based framework to integrate and manage the prosumers in the SG energy sharing process. Please note that some works use the word "community" to indicate the entire prosumer base living in specific region or country; but the concept we introduce in this paper is different from that.

\section{LITERATURE REVIEW}

In this section, we particularly discuss the prosumer participation and management schemes that have been utilized by existing SG energy sharing approaches.

Literature integrates the prosumers to the SG using two main methods; (i) individual integration, and (ii) simple-group integration in the form of a VPP (Virtual Power Plant) (Fig.1). Individual integration method links the individual prosumers to the SG, facilitating the direct energy sharing between the prosumers and utility grid. Here the energy sharing decisions are made based on the individual perceptions. According to the literature, there are several research contributions on SG that consider the individual prosumer participation. For instance, ADDRESS (Active Distribution networks with full integration of Demand and distributed energy RESourceS) [9] is a European initiative that builds a framework to encourage involvement of individual domestic and small commercial users in energy sharing, and builds new relationships between prosumers and utility companies, while investigating real-time price signals and local optimization. Similarly, DR(Demand Response)[10] is a Canadian project, aiming to minimize the energy demand during peak hours using real-time price demand response, while returning the financial incentives to the participants as a return for the commitment to reduce the energy usage. On the other hand, iDEaS project (Intelligent Decentralised Energy-Aware Systems) [11] presents a novel market mechanism and strategies for trading agents to manage the electricity trading within the SG. This trade management considers the impact due to the self-interested participating prosumers and transmission line capacity constraints [12].

Another method is to connect a group of prosumers to the utility grid. When it comes to the energy auctioning, this collaboration becomes a Virtual Power Plant (VPP). Literature recognizes VPP as "a technology for market visibility of many small distributed energy resources" [13]. Integrating group of prosumers attain the critical mass essential to actively involve in energy sharing. As illustrated in Fig. 1, these simple prosumer groups contain different prosumers having diverse behaviors (e.g. different energy sources, different amounts of energy supplied to the grid, etc.), and those prosumers collectively increase the amount of power to be auctioned to the grid. The group of prosumers can attain the minimum amount of energy requested by utility companies with lesser

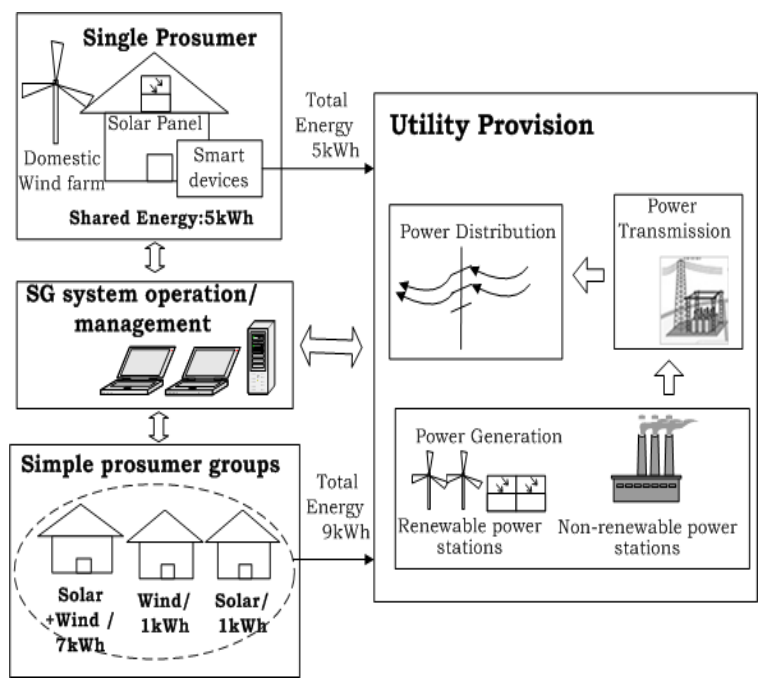

Figure 1. Existing prosumer integration and managing schemes

effort when compared to the individual prosumers. For example, if three prosumers who generate $7 \mathrm{kWh}, 1 \mathrm{kWh}$ and $1 \mathrm{kWh}$ respectively are aggregated into a group, the entire group can offer $9 \mathrm{kWh}$ of collective energy to the grid (Fig. 1). In literature, the VPPs are managed to interact, negotiate or collaborate with other participants of the utility grid to enable joint coordination about the energy usage within the group of houses and thus attain enhanced overall energy efficiency [14]. In literature, we can identify two VPP management architectures, namely centralized architecture and decentralized architecture. In centralized architecture, grid-connected distributed power sources are controlled through a centralized controller [15]. The centralized controller is responsible for capturing power flow information, analyzing, and decision making and controlling the prosumers in VPP. In contrast, in decentralized architecture, the agents can act independently without the involvement of central controller. Decentralized VPP enables the prosumer to autonomously perform certain communications and decision making tasks [16]. In addition, the agent integrated VPPs can communicate with the power market and also can negotiate with other agents that control micro-sources [17].

A combined effort of individual prosumer and group involvement is presented in eTelligence project [18] to diminish the load on the utility grid in the Cuxhaven city and surrounding areas, allowing private prosumers as well as prosumer groups (VPPs) to put any quantities of energy on the market using an automated infrastructure linked to the market structure.

\section{RESEARCH RATIONALE}

Although the literature presents several efforts on prosumer participation and management schemes, many issues are still available that require further attention. In this section we discuss the shortcomings of existing schemes and hence identify the open research issues. 


\section{A. Shortcomings of Existing Literature}

Table. 1 summarizes the shortcomings of existing literature, by checking whether the existing works effectively address the following questions.

Q1. Does the framework support energy sharing between prosumers and the utility grid?

Q2. Does the framework support energy sharing between consumers and prosumers?

Q3. What type of integration is used to connect prosumers to SG: Individual (I), or simple-Group (G) ?

Q4. Does the framework follow a proper method to make prosumer aggregations rather than collecting prosumers in ad-hoc manner?

Q5. Is there an approach to identify negative behaviors of prosumers (e.g. prosumer suddenly decides to supply lesser amount of energy than agreed)?

Q6. Is there an effective method to identify and attract more dynamic prosumers (prosumers capable to offer more energy to the grid) to grow the prosumer-base?

Q7. Is there a comprehensive rewarding scheme that considers both financial and non-financial incentives to prosumers?

\section{B. Open Research Issues}

Based on the above review, following research issues are identified that requires further investigated in future studies.

1) No approaches are found that discuss the energy sharing between the prosumer and the consumer: If consumers purchase energy from geographically closer prosumers without interrupting the main power grid, this will reduce the energy transfer cost, thus reduce the energy loss and ultimately result in efficient energy exchange. In current research field, no research has investigated the energy sharing between prosumers and consumers. However, the energy sharing between the prosumers and consumers is challenging, as in most cases, the individual prosumers are not capable to supply high quantity of energy, hence fail to fulfill even the single consumer's energy requirement. Therefore in most scenarios the prosumer aggregations are necessary to supply higher amount of energy to the consumers. However if these aggregations are not sufficiently stable and sustainable, fulfilling the consumers' energy demand in long-term is in doubt.

2) No approaches are found that follow a proactive method to group the prosumers: It's true that making prosumer-groups can achieve higher amount of energy, hence can accomplish higher market visibility. However the current prosumer-groups are established in ad-hoc manner without following an effective grouping method. In fact, the grouping of prosumers should be done with extreme care considering diverse energy behaviors (such as energy sources and quantity of energy can be shared) of different prosumers. If the prosumers with completely different behaviors and goals are
TABLE I. SHORTCOMINGS OF EXISTING APPROACHES

\begin{tabular}{|c|c|c|c|c|c|c|c|}
\hline Approach (Reference) & $\begin{array}{c}\mathbf{Q} \\
\mathbf{1}\end{array}$ & $\begin{array}{c}\mathbf{Q} \\
\mathbf{2}\end{array}$ & $\begin{array}{c}\mathbf{Q} \\
\mathbf{3}\end{array}$ & $\begin{array}{c}\mathbf{Q} \\
\mathbf{4}\end{array}$ & $\begin{array}{c}\mathbf{Q} \\
\mathbf{5}\end{array}$ & $\begin{array}{c}\mathbf{Q} \\
\mathbf{6}\end{array}$ & $\begin{array}{c}\mathbf{Q} \\
\mathbf{7}\end{array}$ \\
\hline $\begin{array}{c}\text { ADDRESS project } \\
\text { (Belhomme et al. 2008) }\end{array}$ & $\mathrm{Y}$ & $\mathrm{N}$ & $\mathrm{I}$ & - & $\mathrm{N}$ & $\mathrm{N}$ & $\mathrm{N}$ \\
\hline S-TEN Project & $\mathrm{Y}$ & $\mathrm{N}$ & $\mathrm{I}$ & - & $\mathrm{N}$ & $\mathrm{N}$ & $\mathrm{N}$ \\
\hline $\begin{array}{c}\text { iDEaS Project } \\
\text { (Vytelingum et al. 2010) }\end{array}$ & $\mathrm{Y}$ & $\mathrm{N}$ & $\mathrm{I}$ & - & $\mathrm{N}$ & $\mathrm{N}$ & $\mathrm{N}$ \\
\hline $\begin{array}{c}\text { DR Project (Demand } \\
\text { Response 2008) }\end{array}$ & $\mathrm{Y}$ & $\mathrm{N}$ & $\mathrm{I}$ & - & $\mathrm{N}$ & $\mathrm{N}$ & $\mathrm{Y}$ \\
\hline $\begin{array}{c}\text { (Pudjianto, Ramsay, and } \\
\text { Strbac 2007) }\end{array}$ & $\mathrm{Y}$ & $\mathrm{N}$ & $\mathrm{G}$ & $\mathrm{N}$ & $\mathrm{N}$ & $\mathrm{N}$ & $\mathrm{N}$ \\
\hline $\begin{array}{c}\text { Smart House/ Grid } \\
\text { project (Kok et al. 2009) }\end{array}$ & $\mathrm{Y}$ & $\mathrm{N}$ & $\mathrm{G}$ & $\mathrm{N}$ & $\mathrm{N}$ & $\mathrm{N}$ & $\mathrm{N}$ \\
\hline $\begin{array}{c}\text { eTelligence project } \\
\text { (eTelligence 2008) }\end{array}$ & $\mathrm{Y}$ & $\mathrm{N}$ & $\begin{array}{c}\text { G } \\
\text { /I }\end{array}$ & $\mathrm{N}$ & $\mathrm{N}$ & $\mathrm{N}$ & $\mathrm{N}$ \\
\hline $\begin{array}{c}\text { (Dimeas and } \\
\text { Hatziargyriou 2007) }\end{array}$ & $\mathrm{Y}$ & $\mathrm{N}$ & $\mathrm{G}$ & $\mathrm{N}$ & $\mathrm{N}$ & $\mathrm{N}$ & $\mathrm{N}$ \\
\hline $\begin{array}{c}\text { (Chalkiadakis et al. } \\
\text { 2011) }\end{array}$ & $\mathrm{Y}$ & $\mathrm{N}$ & $\mathrm{G}$ & $\mathrm{N}$ & $\mathrm{N}$ & $\mathrm{N}$ & $\mathrm{Y}$ \\
\hline
\end{tabular}

aggregated, it leads to misunderstandings and disputes among prosumers within the same group. This will ultimately result the prosumers to leave their groups. Therefore these simple groups are incapable to sustain in the competitive energy market in long-term.

3) No methods to identify the risks associated with negative behaviours of prosumers: In an effective prosumer management system, it is necessary to identify prosumers' negative behaviours, to analyze the associated vulnerability, and to resolve these negative events to avoid long-term negative effects. However the existing methods are lack of these techniques. In order to explain this issue more comprehensively, we categorize the prosumer behaviours as follows; (i) positive behaviours, (ii) neutral behaviours, and (iii) negative behaviours. Positive behaviour is when the prosumer acts more beneficial manner than what was initially expected to achieve, may be offering more energy to the utility grid than agreed. Neutral behaviour is when a prosumer acts exactly what was expecting to achieve. Negative behaviour is when the prosumer does not act as agreed, may be purposely supplying lower amount of energy than agreed over a long period of time. In addition to these prosumers' activities, prosumers' opinions, future plans and interests can also be positive, negative or neutral. For example, prosumer's interest to store energy for future use rather than sharing with the utility grid can be a negative behaviour with respect to energy sharing aspects; and unfair distribution of financial benefits among the prosumers can make negative opinion about the energy sharing process. These negative events can be a serious threat to the sustenance of energy sharing process, if not resolved efficiently.

4) There is no comprehensive approach to identify and attract more dynamic prosumers to grow the prosumer base: Relying on the minimum number of prosumers is shortsighted in SG energy sharing, due to the uncertainty involved with climate dependant green energy sources and prosumer behaviours. For example, if there is a rainy fortnight that 
avoids the prosumers to generate the sufficient energy using their roof-top solar panels, the minimum number of prosumers may not be able to satisfy even the minimum energy expectations of the utility company. If more prosumers are attracted for energy sharing process, especially the dynamic prosumers who supply higher amount of energy in long-term; the utility company can collect the required amount of energy to fulfill the customer demand. However, in literature, no clear approaches have been presented to identify or attract more dynamic prosumers to grow the prosumer-base.

5) Lack of comprehensive prosumer rewarding schemes that consider both financial and non-financial incentives: Prosumer rewarding schemes play critical role in achieving a motivated prosumer-base. These rewards can be either financial incentives like the monitory value given for the energy generated and supplied to the grid or non-financial rewards such as social respect, reputation and popularity for generating and sharing the green energy. In order to achieve an effective rewarding scheme, both these financial and nonfinancial incentives should be optimally amalgamated and fairly offered to the prosumers. However the existing approaches have not presented comprehensive prosumer rewarding schemes, rather considering only the financial return to the prosumers.

\section{The CONCEPT OF Prosumer-COMMUNITY}

In this section, we introduce a new concept of 'prosumercommunity' as an approach to participate and manage the prosumers in the SG energy sharing process. This method connects the prosumers to the SG in the form of goal-oriented virtual communities. The community of prosumers interacts with each other and with the local distributor or utility grid through the community gateway, which is a smart intermediate and intelligent component that bridges the utility grid with smart devices of each distributed energy resource that belongs to the community (Fig. 2). Further implementation details are out of the scope of this paper.

Before move into the concept of the prosumer-community, we first briefly explain the general aspects behind the virtual community (VC). In literature, the term "Virtual community (VC)" is defined as "a social network of individuals who interact through specific media, potentially crossing geographical and political boundaries in order to pursue mutual interests or goals" [19]. VC aspects have been extensively utilized in diverse areas such as healthcare [20], education [21], peer-to-peer services [22], and social network/blog space communities [23] [24]. However, no research has been carried out to apply community aspects to the SG energy sharing or even to the power networks.

In fact, the prosumer community is a major improvement to the simple prosumer groups. As we discussed in previous sections, simple prosumer groups increase the collective amount of power supplied to the grid by aggregating the prosumers in ad-hoc manner and therefore these groups may contain prosumers having diverse behaviours (e.g. different energy sources, different amounts of energy supplied to the

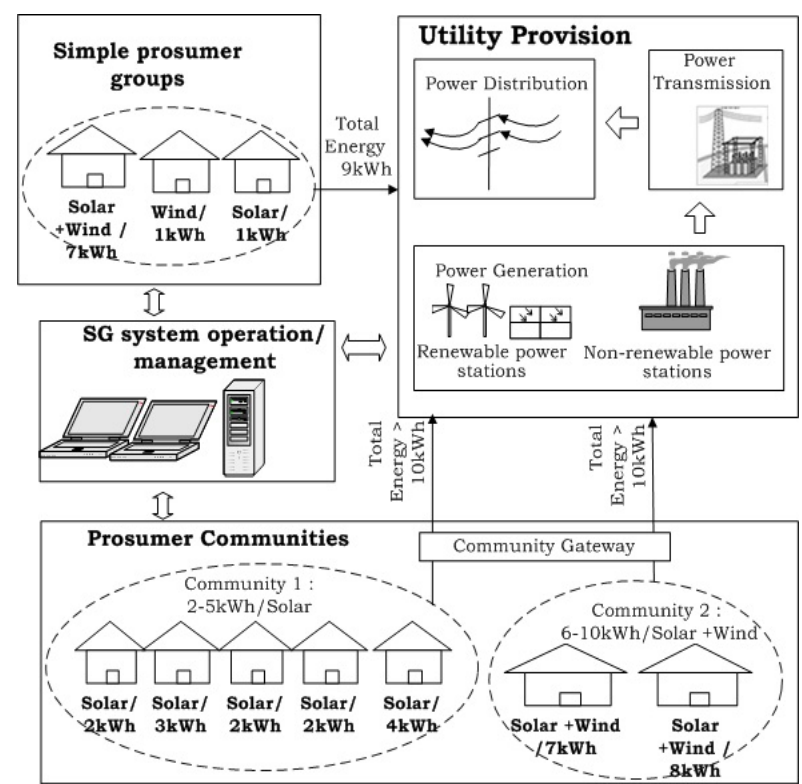

Figure 2. The concept of prosumer community

grid, etc.) (Fig. 2). In addition, these groups are not provoked to achieve a predefined goal. Therefore in some cases, those simple groups may fail even to achieve the minimum energy threshold accepted by the utility company. On the other hand, compatible to the known definition of $\mathrm{VC}$, the prosumercommunities are established aggregating the prosumers from different locations, but having similar interests and behaviours (e.g. similar energy sources, similar amount of energy supplied the grid, etc.). Those communities are stimulated to achieve a predefined common goal (e.g. to achieve at least the minimum amount of energy requested by utility provision, the minimum amount of energy required to obtain higher bargaining power in the energy market, etc.). One way to obtain prosumercommunities can be illustrated using Fig.2. In Fig. 2, the prosumers who offer energy between $2-5 \mathrm{kWh}$ through their domestic solar panels will be added to one community and the prosumers who offer energy between $6-10 \mathrm{kWh}$ will be added to another community. The communities are provoked to achieve minimum energy of $10 \mathrm{kWh}$, which is the community goal and the number of members of the communities is varied accordingly.

As all the other general community based approaches, the prosumer-communities also contain three main processes namely prosumer-community formation, prosumer community growth and prosumer-community overall management. Although the prosumer-communities inherit more sustainable characteristics compared to the existing approaches, the real challenge is developing smart methods for these three processes, while addressing the research issues identified in section III. In next sub-section, we suggest subtasks for each of these processes to achieve optimal prosumer-communities. 


\section{A. Prosumer-Community Architecture}

Fig. 3 illustrates the subtasks associated with prosumercommunity formation, prosumer-community growth and prosumer-community overall management. These processes are further analyzed as follows;

1) Prosumer- community formation: Forming a prosumer community is not a simple task. Unlike the most existing virtual communities, the membership of prosumer community is limited to a set of individuals having energy resources and smart infrastructures to communicate with the SG. The formation also can be a long and complicated process that involves with interacting different authorized agents. As the initial step, the households' energy behaviours that affect the energy sharing process should be captured either explicitly by direct contact of prosumers or implicitly by observing the smart meters in long term. Some of the many quantitative energy behaviours are average daily power generation and consumption, capacity of smart storage, expected power variation due to the future plans, seasonal power variation (green power variation due to climate change), prosumer's preferred quantity of energy for future consumption, prosumer's preferred quantity of energy for sharing and prosumers' preferred level of feed-in tariff, etc. After analysing the diverse energy behaviours of prosumers, the prosumers having common interests should be segmented to form the communities. Defining community goals consider many influential parameters in addition to the prosumers' energy behaviours. Some of those parameters are current energy buyers, current rate of power in competitive power market, pricing policies, minimum and maximum amount of collective power to be achieved per transaction with the utility grid and the maximum and minimum number of prosumers in one community to achieve sustainable quantity of power, etc. Different communities are characterized with different community goals and prosumers are attached to the most appropriate community. Since the community formation involves with a complicated process, a comprehensive bootstrapping process is necessary for the prosumers waiting to join the community to discover community regulations such as community pre-qualification criteria, community goals, and other rules and regulations associated with incentive distributions, deactivations, breach of contracts, etc.

2) Prosumer community growth: As we discussed earlier, relying on existing number of members is very short sighted in energy sharing process. Therefore it is necessary to grow the Prosumer-communities continuously to achieve long-term stability. The promotion of the prosumer-communities to attract more members should be done through a responsible body (e.g. utility company), by giving clear presentation of rules and regulation, and pre-qualification criteria. In community growth, it is necessary to analyse historic energy profile of prosumers and identify more dynamic prosumers who can provide higher amount of energy for long period of time. To stimulate more dynamic members to join the prosumer-communities, the strategies like increasing the

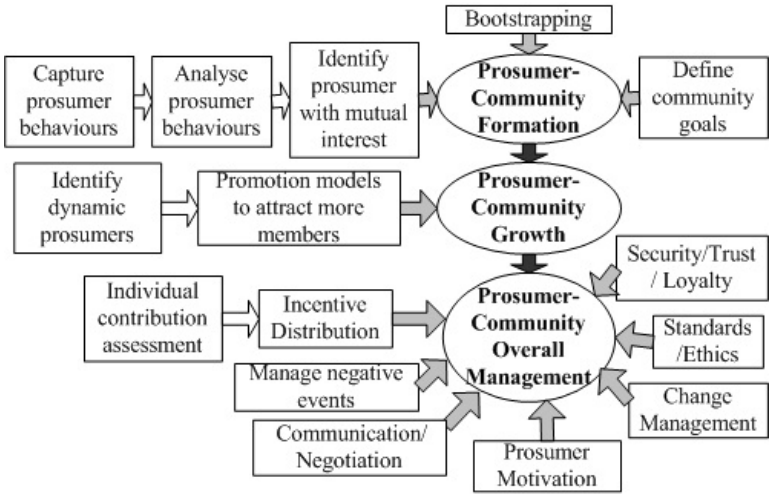

Figure 3. Prosumer-community formation growth and management

feed-in tariff for the prosumers who would agree higher energy contribution, are possible.

3) Prosumer-community overall management: As the prosumer-communities grow with new members, the challenges involved with making these communities sustainable also will increase. The community is said to be sustainable if it exhibits an intrinsic prolonged existence over long duration, perhaps over generations [25]. The main factors that determine the sustainability in prosumer-community management schemes are; proactive individual contribution assessment schemes followed by fair distribution of rewards, effective management of negative events, prosumer motivation schemes, effective communication and negotiation schemes, ability to withstand radical changes over time, clear presentation of ethics and standards, and security, trust and loyalty within the community. Most of these factors are interdependant, for example, to motivate the prosumers and to stimulate prosumers' positive behaviours, it is necessary to distribute the rewards fairly among the community members based on the relative performances of each prosumer considering the energy contribution he made and the duration of his active membership. On the other hand, if one prosumer acts negatively, it may adversely affect the incentives attained by all the members in the entire prosumer-community. This will ultimately create disputes among the members, reduce the trust among members and make some prosumers to leave their communities. Therefore risk identification, assessment and resolution schemes are really necessary. In addition the community goals should be defined based on the changes in energy market and the members should be empowered to accept the changes.

\section{B. Significance of Optimal Prosumer-Community}

Enabling optimal-prosumer communities offers numerous socio-economic benefits. Some of the many benefits are illustrated as follows;

1) Enhances the prosumers' bargaining power in longterm: Since the prosumer-community can produce higher quantity of collective energy in long-term compared to the 
existing approaches, the communities can offer higher bargaining power for its members in long-term.

2) Achieves higher sustainability: The members of each community exhibit similar behaviours. This makes incentive distribution and maintenance process easier, and reduces contradictions among members, hence leads to sustainable communities.

3) Prosumer-communities' capability to fulfill consumers' energy demand results efficient energy transfer, reduced energy transfer cost and reduced energy loss: The consumers can trust more on sustainable prosumer-communities in longterm than the individual prosumers or simple prosumer groups in purchasing the energy. Therefore the consumers can directly request the energy from geographically closer prosumer-community. This makes the energy transfer distance shorter, achieving more efficient energy transfer, and also reduces the energy transfer cost and energy transfer loss.

4) Make electricity user an active part of electricity value chain: Prosumer-communities achieve long-lasting market visibility for a individual prosumer. This creates a strong interaction among the prosumers and also between the prosumer and the utility grid in long-term, which is critical in remote areas as all individuals have to work together efficiently to manage their energy usage, energy requirement and energy generation.

\section{CONCLUSIONS AND FUTURE WORK}

In this paper, we extensively analyze the prosumer participation and management schemes presented in existing energy sharing approaches and highlight the open research issues. Accordingly, it is evident that most of the previous contributions in the field of SG energy sharing have overlooked the area of managing prosumers. In order to achieve more sustainable prosumer participation and management scheme, we suggest a novel concept of goaloriented virtual prosumer-communities. We identify the challenging tasks that should be accomplished in prosumercommunity formation, growth and management processes. In future research steps, we will develop smart techniques to form, grow and manage the optimal prosumer communities and endeavor to resolve the identified shortcomings in existing literature.

\section{ACKNOWLEDGMENT}

This work is supported by the Australian Research Council Linkage, project ID: LP100200693.

\section{REFERENCES}

[1] US department of energy, "International Energy Outlook 2011", http://www.eia.doe.gov/oiaf/ieo/world.html, 2011, (05/04/2011).

[2] "Customer Led Network Evolution", http:/www.networkrevolution.co.uk/whyarewedoingthis/abouttheproject /whatisasmartgrid, (02/04/2011).

[3] T. S. Dillon, A. Talevski, V. Potdar and E.Chang, "Web of Things as a Framework for Ubiquitous Intelligence and Computing," in Proceedings of the 6th International Conference on Ubiquitous Intelligence and Computing, Brisbane, Australia, 2009, pp. 2-13.

[4] J. A. Momoh, "Smart grid design for efficient and flexible power networks operation and control." pp. 1-8.
[5] A.J. D. Rathnayaka, V. M. Potdar, and S.Kuruppu, "Evaluation of Wireless Home Automation Technologies," in 5th IEEE International Conference on Digital Ecosystems and Technologies, Korea, 2011.

[6] C. Cecati, G. Mokryani, A. Piccolo and P. Siano, "An overview on the smart grid concept", Proc. 36th Annual Conference on IEEE Industrial Electronics Society(IECON 2010), 2010, pp. 3322-3327

[7] V. C. Gungor, L. Bin, and G. P. Hancke, "Opportunities and Challenges of Wireless Sensor Networks in Smart Grid," Industrial Electronics, IEEE Transactions on, vol. 57, no. 10, pp. 3557-3564, 2010.

[8] V. K. Sood, D. Fischer, J. M. Eklund et al., "Developing a communication infrastructure for the Smart Grid." pp. 1-7.

[9] R. Belhomme, R. C. R. D. Asua, G. Valtorta, A. Paice, F. Bouffard, R. Rooth, et al., "ADDRESS - active demand for the smart grids of the future," IET Seminar Digests, vol. 2008, no. 12380, pp. 33, 2008.

[10] Independent Electricity System Operator, "Demand Response Project," http://www.ieso.ca/imoweb/consult/demandresponse.asp, (30/03/2011)

[11] "iDEaS: Intelligent Decentralised Energy-Aware Systems," http://www.ideasproject.info/index.php. (05/04/2011)

[12] P. Vytelingum, T.D.Voice, S.D.Ramchurn, A.Rogers and N.R.Jennings, "Agent-Based Micro-Storage Management for the Smart Grid," in The 9th International Conference on Autonomous Agents and Multiagent Systems (AAMAS 2010), Toronto, Canada, 2010, pp. 39-46.

[13] Mihai, "Virtual power plants: A solutionfor diminishing the barriers for the penetration of uncontrollable resources", in Energy Infrastructure Scenarios for the South-Eastern Europe, pp. 1-13, SUSPLAN-Regional Kick-Off Workshop, 2009.

[14] K. Kok, S. Karnouskos, D. Nestle, A. Dimeas, A. Weidlich, C. Warmer et al., "Smart houses for a smart grid" , n Electricity Distribution - Part 1, 2009, pp. 1-4.

[15] D. Pudjianto, C. Ramsay, and G. Strbac, "Virtual power plant and system integration of distributed energy resources," IET Renewable Power Generation, vol. 1, no. 1, pp. 10-16, 2007.

[16] G. Chalkiadakis, V. Robu, R. Kota, A. Rogers and N. Jennings, "Cooperatives of Distributed Energy Resources for Efficient Virtual Power Plants," in The Tenth International Conference on Autonomous Agents and Multiagent Systems (AAMAS-2011), Taipei, Taiwan, 2011.

[17] A. L. Dimeas, and N. D. Hatziargyriou, "Agent based control of Virtual Power Plants," in International Conference on Intelligent Systems Applications to Power Systems, 2007. ISAP 2007, 2007, pp. 1-6.

[18] "Project Summary: eTelligence", 2008 http://etelligence.de/etelligence.php, (30/03/2011).

[19] "Virtual community," http://glossary.assistnz.com/glossarylist/virtualcommunity, (1/4/2011).

[20] G. Demiris, "Virtual Communities in Health Care," Intelligent Paradigms for Healthcare Enterprises, Studies in Fuzziness and Soft Computing B. G. Silverman, A. Jain, A. Ichalkaranje et al., eds., pp. 5858: Springer Berlin / Heidelberg, 2005.

[21] M. Parsell, and J. Duke-Yonge, "Virtual communities of enquiry : an argument for their necessity and advice for their creation," E-Learning and Digital Media, vol. 4, no. 2, pp. 181-193, 2007.

[22] I. Radovanović, J. Lukkien, S. Chen, C. Molanus and T. Özçelebi, "Virtual community management for enabling $p 2 p$ services in the ims network", in International Conference on Internet Multimedia Services Architecture and Applications, 2008.

[23] L. Backstrom, D. Huttenlocher, J. Kleinberg and X. Lan, "Group formation in large social networks: membership, growth, and evolution,' in Proceedings of the 12th ACM SIGKDD international conference on Knowledge discovery and data mining, Philadelphia, PA, USA, 2006, pp. 44-54.

[24] S.-C. Baek, S. Kang, H. Noh and S.-W. Kim, "Contents-Based Analysis of Community Formation and Evolution in Blogspace," in Proceedings of the 2009 IEEE International Conference on Data Engineering, 2009 pp. 1607-1610.

[25] J. Porra, and M. Parks, "Sustainable virtual communities: suggestions from the colonial model," Information Systems and E-Business Management, vol. 4, no. 4, pp. 309-341, 2006. 